\title{
DER SHIFTA-KRIEG IN KENIA
}

\author{
Von Volker Matthies
}

Von 1963 bis 1967 wurde in Kenia zwischen der Regierung des Landes und aufständischen Somali-Guerillas, von dieser geringschätzig „sh:؟ta"** (Räuber, Banditen) genannt, ein erbitterter Kleinkrieg ausgetragen, der zahlreiche Menschenopfer forderte und erhebliche materielle und finanzielle Verluste mit sich brachte. Von der Mehrheit der Bevölkerung im Lande, den zahlreichen Kenia-Touristen und der Weltöffentlichkeit kaum beachtet, war dieser Krieg einer der zahlreichen „vergessenen Kriege“, wie sie sich seit der Dekolonisation in vielen Ländern der Dritten Welt ereigneten. Während die Regierung des wachsenden Tourismus und ausländischer Investitionen wegen bemüht war, Kenia das Image eines friedlichen, stabilen und prosperierenden Landes zu verschaffen, befand sich etwa ein Drittel des Landes seit der Unabhängigkeit Ende 1963 faktisch im Kriegszustand.

Zwei Faktoren insbesondere trugen dazu bei, den shifta-Krieg zu einem „vergessenen Krieg“ zu machen. Zum einen war dies die restriktive Informationspolitik der Kenia-Regierung, die aus den oben erwähnten Gründen kaum ein Interesse an Publizität über diesen Krieg haben konnte und die in einer Art von Zweckoptimismus dazu neigte, die Vorgänge in den von Somali bewohnten Teilen des Landes zu bagatellisieren. Erst im Jahre 1967, als sich der Krieg durch die zunehmende Intervention Somalias zu einer ernsthaften Bedrohung entwickelte, sah sich die Regierung gezwungen, die Weltöffentlichkeit $\mathrm{zu}$ informieren ${ }^{1}$. Zum anderen war es die isolierte Lage des Kriegsschauplatzes im ariden und menschenarmen Norden und Nordosten des Landes, Gebieten, die kommunikativ kaum in engerer Verbindung mit den politischen, wirtschaftlichen und kulturellen Zentren im Süden Kenias standen.

Von der Wissenschaft wurde dieser Krieg bisher nicht so sehr als ein innerer Krieg Kenias thematisiert, sondern eher als ein Krieg zwischen Kenia und der Republik Somalia, also als ein internationaler Krieg zwischen souveränen Staaten.

So klassifizierte z. B. Istvan Kende in seiner empirischen Untersuchung der Kriege seit Ende des Zweiten Weltkrieges den shifta-Krieg als einen „international or frontier war"2. Desgleichen ordnete auch Evan Luard in seiner Klassifizierung der sechzig wichtigsten Kriege seit 1945 den shifta-Krieg als einen Grenzkrieg zwischen zwei souveränen Staaten ein ${ }^{3}$. Sicherlich haben beide Autoren bei dieser

\footnotetext{
:Die kenianische und äthiopische Regierung benutzten das Wort „shifta“ zur Bezeichnung der aufständischen Somali in ihren Ländern. Das Wort hatte für sie eine negative Bedeutung im Sinne von Banditen und Gcsetzlosen. Aus diesem Grunde wurde es auch von den Somali selbst abgelehnt. Der Botschafter der Republik Somalia in Äthiopien protestierte gegen die Verwendung des Wortes "shifta“ zur Bezeichnung der gegen die kenianische und äthiopische Regierung kämpfenden Somali und teilte mit, die Somali würden es als feindselige Propaganda betrachten (vgl. Africa Report, April 1967, S. 24/25). Die Somali selbst zogen vor, sich "Mudjahaddin“ (Krieger) oder "nationalists“ bzw. "freedom/liberation fighters" zu nennen. Die Herkunft und die Bedeutung des Wortes "shifta“ sind umstritten. Mit großer fighters" zu nennen. Die Herkunft und die Bedeutung des Wortes „shifta sind umstritten. Mit großer
Wahrscheinlichkeit entstammt das Wort der amharischen Sprache und ist vom Verb "sheffete (säffätä) abgeleitet, welches die Bedeutung von rebellieren und des Mißachtens von Recht und Ordnung hat. (Für Hinweise ist der Verfasser Herrn Girma Beshah, Lektor für Amharisch an der Universität Hamburg, dankbar.) Die Herkunft von dem englischen Wort „shifter“ (etwa Schlaukopf, Fuchs) ist unwahrscheinlich; die Kenianer haben das Wort shifta" eher von den Äthiopiern übernommen als von den Engländern (vgl. Doob, Leonard W., Resolving Conflict in Africa, New Haven und London 1970, S. 6). Als Terminus technicus zur Kennzeichnung der Somali-Guerillas in Kenia und Äthiopien hat das Wort weite Verbrcitung gefunden. In diesem Aufsatz wird es nicht in einem abwertenden Sinne benutzt.

1 Kenya-Somalia-Relations, Nairobi 1967.

2 Kende Istvan, Twenty-Five Years of Local Wars, in: Journal of Peace Research, Bd 8, 1971, S. 5-22,

3 Luard, Evan, Frontier Disputes in Modern International Relations, in: Luard, Evan (Ed), The International Reg:lation of Frontier Disputes, London 1970, S. 8.
} 
Einordnung den sog. Grenzkonflikt zwischen Kenia und Somalia und die Intervention Somalias zugunsten der shifta-Guerillas im Blickfeld. Dieser Grenzkonflikt hat seine Wurzeln in dem Somali-Nationalismus, der sich auf eine für Afrika einzigartige ethnisch-kulturelle Homogenität der Somali-Stämme gründet und der sich in der Reaktion auf die kolonialzeitliche Teilung und Penetration der Somali-Stämme durch Großbritannien, Italien, Frankreich und Äthiopien entwickelte. Als sein wichtigstes Ziel verfolgte dieser Nationalismus die Vereinigung aller Somali in einem Staat. Seitdem im Jahre 1960 aus dem Zusammenschluß des ehemaligen britischen Protektorats Somaliland und des ehemaligen italienischen Treuhandgebiets Somalia die Republik Somalia entstand, verfolgte dieser Staat gegenüber seinen starke Somali-Minoritäten beherbergenden Nachbarstaaten Kenia, Äthiopien und Französisch-Somaliland eine pan-somalische und irredentistische Politik. Während diese Politik letztlich auf eine Revision der aus der Kolonialzeit stammenden Grenzen und auf eine Neuziehung der Grenzen nach ethnisch-kulturellen Kriterien hinauslief, hielten Somalias Nachbarstaaten an der kolonialzeitlichen Fixierung der Grenzen und damit am territorialen status quo in Nordostafrika fest und sahen in der Politik Somalias eine Bedrohung ihrer territorialen Integrität und staatlichen Souveränität4. Da über diese konfligierenden Zielsetzungen auf friedlichem Wege keine Einigung erzielt werden konnte, bediente sich die Republik Somalia zur Erreichung ihrer Ziele auch einer quasi-militärischen Intervention ${ }^{5}$. Ohne Zweifel stellen dieser zwischenstaatliche Konflikt sowie die Intervention Somalias zugunsten der shifta-Guerillas in Kenia als exogene Faktoren für das Verständnis des shifta-Krieges sehr wesentliche Determinanten dar; ohne eine Berücksichtigung der endogenen Faktoren jedoch läßt sich nach unserer Meinung eine Analyse dieses Krieges nur mit verstellten Perspektiven durchführen.

Die Kennzeichnung des Konflikts zwischen Kenia und Somalia als "Grenzkonflikt" scheint uns wenig aussagekräftig, ja sogar etwas irreführend zu sein, da sie suggeriert, es handele sich hier ausschließlich um eine Auseinandersetzung zwischen souveränen Staaten um den Verlauf von Grenzen. Bei einer oberflächlichen Betrachtungsweise geht es bei diesem Konflikt sicherlich um eine Adjustierung von Grenzen und die Interpretation von kolonialzeitlichen Grenzverträgen; doch ist der Streit um die Grenzen Ursache oder nur Symptom des Konflikts? Eine tiefergehende Analyse gibt uns den Blick frei auf die ganze Komplexität des Konflikts und führt uns hin zu Problemen der internen politischen und sozialen Struktur der beteiligten Staaten, insbesondere zu Problemen des Nationalismus, der Nationenbildung und ethnischer Minoritäten ${ }^{6}$. Betrachten wir den shifta-Krieg in Kenia

\footnotetext{
$4 \mathrm{Zu}$ diesem Konflikt und zum Somali-Nationalismus siehe vor allem Drysdale, John, The Somali Dispute, London und Uunmow 1964; Touval, Saadia, Somali Nationalism, Cambridge, Mass., 1963; Hoskyns, Catherine (Ed), The Ethiopia-Somali-Kenya Dispute 1960-1967, Dar es salaam, Nairobi, Addis Ábeba 1969; vgl. auch vom Verfasser: Der Grenzkonflikt zwischen Somalia, Äthiopien und Kenia-Penetrationsprozesse, Abhängigkeitsstrukturen und Gegenstrategien in Nordostafrika, ein Beitrag zu dem Sektionsprojekt über Konflikt und Integration der Sektion Internationale Politik der Deutschen Vereinigung für Politische Wissenschaft, i. E.

5 Zum Interventionsproblem in den zwischenstaatlichen Beziehungen Afrikas im allgemeinen und zur Intervention Somalias in seinen Nachbarstaaten im besonderen, vgl. Matthews, Robert O., Domestic and Interstate Conflict in Africa, in: International Journal, vol. 25, 1969/70, S. 463 ff. und 468. Nach seiner Definition umfaßt der Begrifí "Intervention“ ",any organized activity on the part of one state which seeks to undermme, alter, or replace the authority structure of another state (the target)“. Die Intervention Somalias in Kenia umfaßte die Bereitstellung von Operationsbasen, Waffen, Geldmitteln und "Freiwilligen" tür die shifta. Ergänzend könnte man noch auf die propagandistische. Unterstützung durch Radio-Propaganda verweisen, vgl. dazu Legum, Colin, Somali Liberation Songs, in: Journal of Modern African Studies, vol. 1, No. 4, März 1963, S. 503-519; zu den Techniken zwischenstaatlicher Intervention in Afrika vgl. auch Zartman, I. William, International Relations in the New Africa, Englewood Cliffs, N. J., 1966, S. 88.

$6 \mathrm{Vgl}$. Tägil, Sven, The Study of Boundaries and Boundary Disputes, in: Widstrand, Carl Gösta (Ed)

African Boundary Problems, Uppsala 1969, S. 22-32; vgl. im gleichen Band auch Widstrand, C. G., Some African Boundary Problems: A Discussion, S. 168-171.
} 
vor diesem Problemhorizont, so scheint er uns eher ein innerer Krieg als ein internationaler Krieg zu sein, wobei wir uns allerdings der Problematik der theoretischen und praktischen Abgrenzung zwischen beiden Kriegstypen bewußt sind.

Wie die wissenschaftliche Diskussion um das Phänomen des „internal war" und dessen internationale Aspekte gezeigt hat ${ }^{7}$, ist eine strikte Unterscheidung des inneren vom internationalen Krieg kaum möglich, da fast jeder innere Krieg auch internationale Elemente enthält. Zudem bereitet die Bestimmung des Bezugssystems des „internal war“ erhebliche Schwierigkeiten. Eine Bestimmung nach den formalen Kriterien des Völkerrechts allein, nach der alle solche Kriege innere Kriege sind, die sich innerhalb von der Völkerrechtsgemeinschaft anerkannter souveräner Staaten ereignen, scheint unzureichend $z u$ sein, da viele Staaten keine einheitliche politische Kultur besitzen und oftmals Teile ihrer Staatsbürger transnationalen Loyalitäten anhängen: „What appears internal from the legal point of view ... may be external from the cultural point of view ..."8. Wie noch zu zeigen sein wird, trifft genau dieses Problem auf den shifta-Krieg zu: nach formaljuristischen Kriterien waren die Somali zwar Bürger Kenias, nach der Orientierung ihrer politischen Kultur, nationalen Loyalitäten und Identifikationen jedoch fühlten sie sich eher der Republik Somalia zugehörig. Nach K. W. Deutsch sind in den meisten inneren Kriegen „elements of domestic strife and of external intervention in varying proportions" miteinander vermischt; echte innere Kriege („authentic internal wars") unterscheiden sich dabei von Stellvertreterkriegen („wars by proxy“) durch ein eindeutiges Übergewicht interner Elemente 9 . So gewinnt das Ausmaß einer externen Intervention eine große Bedeutung bei der Bestimmung von inneren Kriegen; jedoch ist Deutsch gegenüber der Ansicht von Eckstein zuzustimmen, daß selbst bei einer massiven äußeren Intervention die Authentizität eines inneren Krieges nicht notwendigerweise in Frage gestellt sein muß ${ }^{10 !}$ Letztlich scheint die Entscheidung bei der Bestimmung eines Krieges als „internal“ eine Entscheidung nach Evidenzkriterien und nach dem jeweiligen Focus der Analyse zu sein. In diesem Sinne verwenden wir hier den Begriff „internal war" als Arbeitskategorie, um ganz spezifische Aspekte des shifta-Krieges zu erörtern. Wir betrachten den shifta-Krieg also nicht mehr als einen internationalen Krieg mit internen Implikationen, dessen Akteure souveräne Staaten sind, sondern als einen inneren Krieg mit externen Implikationen, dessen Akteure die Regierung eines souveränen Staates und eine Bevölkerungsgruppe dieses Staates sind ${ }^{11}$. Innerhalb der Klassifikation von Kende bezeichnen wir dabei den shifta-Krieg nicht mehr als einen „international or frontier war", sondern als einen inneren Krieg vom Typ eines "tribal war“ und thematisieren ihn damit als einen Kriegstyp, der

7 Vgl. vor allem Eckstein, Harry (Ed), Internal War, London 1964 und Rosenau, James (Ed), International Aspects of Civil Strife, Princeton, N. J., 1964; auf die neuere Literatur sei hier nur exemplarisch hingewiesen: Kelly, G. A. und Miller, L. B., Internal War and International Systems, Cambridge, Mass. 1969; Mitcheli, C. R., Civil Strife and the Involvement of External Parties, in: International Studies Quarterly, vol. 14, No. 2, 1970, S. 166-194; Bull, Headly, Civil Violence and International Order, in: Adelphi Papers, Nr. 83, London 1971, S. 27-36.

8 Eckstein, Harry, Introduction. Toward the Theoretical Study of Internal War, in: Eckstein (Anmerkung 7), S. 15.

9 Deutsch, Karl W., External Involvement in Internal War, in: Eckstein (Anmerkung 7), S. 102. Ein "authentic internal war" ist nach Deutsch ein innerer Krieg, wenn "on both sides of such a conflict, there is a clear quantitative proponderance of domestic motivations, recruitment and resources". "If outside manpower, motives, money, and other resources appear to constitute the main capabilities committed to the struggle on both sides, then we are inclined to speak of a war by proxy"..."

10 Eckstein, Introduction (Anmerkung 8), S. 15.

11 Zur Kritik am Konzept der. Souverünität und der langjährigen Ǔbung in der Lehre von den Internationalen Beziehungen, souverüne Staaten als die primären, wenn nicht sogar die einzigen internationalen Akteure zu betrachten, vgl. Rosenau, James, Introduction, in: Rosenau (Anmerkung 7), S. 3. 
nach Kende charakteristisch für die jungen unabhängigen Staaten der Dritten Welt geworden ist ${ }^{12}$.

Zunächst wollen wir die Genesis und Ätiologie des shifta-Krieges skizzieren, dann seinen Verlauf und seine politischen Implikationen für Kenia erörtern und abschließend einige Betrachtungen $\mathrm{zu}$ den Problemen und Chancen einer dauerhaften Friedenslösung anstellen ${ }^{13}$.

\section{Zur Genesis und Æ̈tiologie des shifta-Krieges ${ }^{14}$}

Während der britischen Kolonialherrschaft in Kenia wurden die Somali zusammen mit anderen Bevölkerungsgruppen (Galla, Rendille u. a.) innerhalb des „Northern Frontier District“ (NFD), im Norden Kenias zur Grenze mit Äthiopien und Italienisch-Somalia (dem späteren südlichen Landesteil der Republik Somalia) hin gelegen, verwaltet ${ }^{15}$. Die überwiegende Mehrheit der Somali war in dieses Gebiet im Gefolge nomadischer Wanderungen erst nach 1860 eingedrungen. Diese Wanderungen waren begleitet von Kämpfen der Somali untereinander und von Kämpfen der Somali gegen andere Bevölkerungsgruppen. Erst um 1919 gelang den Briten (seit 1896 Formierung des British East Africa Protectorate) eine erste, wenn auch noch immer prekäre Pazifierung der Somali.

Innerhalb des NFD (102000 Quadratmeilen) mit seiner Gesamtbevölkerung von etwa 280000 (200000-390 000) Menschen bewohnten die Somali als größte Bevölkerungsgruppe (etwa 200 000) vor allem den östlichen Teil des NFD (Sub-Distrikte: Garissa, Wajir, Mandera). Die wichtigsten im NFD vertretenen Clan-Familien der Somali waren die Darod (mit dem Sub-Clan der Ogaden) und die Hawiye (mit den Sub-Clans der Ajuran, Degodia, Murille und Gurre). Die nicht-somalischen Bevölkerungsgruppen im NFD, von denen zahlenmäßig die Galla am bedeutendsten waren (80000), lebten mehr im Norden und Nordwesten des Distrikts (Sub-Distrikte: Marsabit, Isiolo, Garissa) ${ }^{\mathbf{1 6}}$.

Der NFD war ein überwiegend arides Steppen- und Savannengebiet, das nur an wenigen begünstigten Stellen (etwa um Marsabit und Moyale) Ackerbau ermöglichte, und unterschied sich aufgrund dieser geographischen und klimatischen Faktoren grundlegend von den überwiegend fruchtbaren Gebieten im Süden Kenias ${ }^{17}$. Aufgrund dieser natürlichen Gegebenheiten war im NFD nur eine nomadische Weidewirtschaft möglich, die sowohl von den Somali als auch von den meisten anderen Bevölkerungsgruppen im NFD betrieben wurde. Ethnisch-kulturell und

12 Kende (Anmerkung 2) unterscheidet zwei Haupttypen von inneren Kriegen; den "anti-regime war" (= "with the aim of overthrowing the central government and changing the regime itself") und den "tribal war" (= outcome of separationist, secessionist, minority, tribal or religious, and similar "conflicts"), S. 11 und S. 13: "tribal wars' become the wars of the newly independent countries".

13 Dies alles muß verständlicherweise mit der im Rahmen eines Aufsatzes gebotenen Kürze geschehen; die hier behandelte Thematik und Problematik ist Teil einer Dissertation über den Grenzkonflikt zwischen Somalia, Athiopien und Kenia, an der der Verfasser zur Zeit arbeitet.

14 Dieser Abschnitt basiert hauptsächlich auf folgenden Werken: Lewis, I. M. The Problem of the Northern Frontier District of Kenya, in Race, vol, 5, 1963, S. 48-60- ders., The Modern History of Somaliland, London 1965, S. 183-195; Castagno, A. A., The Somali-Kenyan Controversy: Implications for the Future, in: Journal of Modern African Studies, vol. 2, No. 2, 1964, S. 165-188; McEwen, A. C., International Boundaries of East Africa, Oxford 1971, S. 113-128; Melamid, Alexander, The Kenya-Somalia Boundary Dispute, in: Geographical Review, vol. 54, No. 4, 1964, S. 586-588; Turton, E. R., Somali Resistance to Colonial Rule and the Development of Somali Political Activity in Kenya 18931960, in: Journal of African History, vol. 13, No. 1, 1972, S. 117-143; Drysdale (Anmerkung 4), Kap. 2, 9-13, 15; Touval (Anmerkuing 4),Kap. 12.

15 Zur Grenzzichung, vgl. vor allem McEwen und Melamid (Anmerkung 14).

$16 \mathrm{Zu}$ den Zahlenangaben, die auf Schätzungen beruhen und für den Zeitraum um 1960 gelten, vgl. Lewis, Race (Anmerkung 14), S. 48, 51 und Castagno (Anmerkung 14), S. 167.

$17 \mathrm{Vgl}$. dazu Ominde, S. H., The Semi-Arid and Arid Lands of Kenya, in: Ominde, S. H. (Ed), Studies in East African Geography and Development, London, Nairobi, Ibadan 1971, S. 146-161. 
sozial waren die Nomaden des NFD ebenfalls grundlegend von den seßhaften Ackerbauern im Süden Kenias unterschieden. Sie gehörten der hamitischen Völkerfamilie und der kuschitischen Sprachfamilie an, waren zum großen Teil Moslems (alle Somali und Teile der Galla), und zeichneten sich gegenüber den mehr hierarchischen Sozialstrukturen der Bantu und Niloto-Hamiten im übrigen Kenia durch ihre segmentär-demokratischen Sozialstrukturen aus.

Eine wesentliche Folge dieser geographischen und ethnisch-kulturellen Besonderheiten des NFD, die von der britischen Kolonialverwaltung erkannt wurden, war, daß der NFD einen besonderen Verwaltungsstatus erhielt und unter Anwendung der "Outlying Districts Ordinance" als ein "Closed District" (seit 1926) verwaltet wurde. $\mathrm{Da}$ scharfe Restriktionen für Reisen in und aus dem Distrikt bestanden und zudem im Distrikt andere Gesetze und Verwaltungstechniken angewandt wurden als im übrigen Kenia, war eine Kommunikation zwischen der Bevölkerung des NFD und des übrigen Kenia kaum möglich. Diese Art der Verwaltung des NFD, die Lewis nicht ganz zu Unrecht als eine Art „holding operation“ zur Abdämmung des südlichen Expansionsdranges der NFD-Nomaden und zur Aufrechterhaltung von Ruhe und Ordnung innerhalb des Distrikts bezeichnete ${ }^{18}$, war verständlicherweise der sozio-ökonomischen und kulturellen sowie politischen Entwicklung des NFD nicht gerade förderlich. Von der personell stets unterbesetzten und nicht immer qualifizierten Administration des NFD wurde kaum etwas getan, um die anhaltende Bodenerosion $\mathrm{zu}$ bekämpfen und die Wasserversorgung zu verbessern. Infolge von Überweidung und Wassermangel kam es im NFD immer wieder zu Unruhen und Stammeskämpfen. Es fehlte im NFD an Straßen, an Schulen und an medizinischen und sozialen Versorgungseinrichtungen. Während im Süden Kenias die Urbanisierung voranschritt und es zu modernen ökonomischen, sozialen und politischen Entwicklungsprozessen kam, wurde die Urbanisierung im NFD von der Kolonialverwaltung bewußt behindert und keine den modernen Entwicklungsprozessen im Süden vergleichbare Entwicklung induziert. Die ungünstigen geographisch-klimatischen Verhältnisse und mangelnden ökonomischen Ressourcen des NFD schienen der Kolonialverwaltung Grund genug, den Distrikt gegenüber den südlichen Landesteilen Kenias zu vernachlässigen. So entwickelte sich der NFD im Lauf der Jahre gegenüber dem südlichen „Zentrum“ Kenias immer mehr $\mathrm{zu}$ einer marginalisierten „Peripherie"19: nach Lewis war der NFD „the most isolated, and the most backward portion of Kenya" und der wohl rückständigste Teil aller Somali-Gebiete im Horn von Afrika ${ }^{20}$, und nach dem Urteil von Soja war er zur Zeit der Unabhängigkeit Kenias „,beyond effective national control and functionally not part of the Kenya nation"21.

Politisch verhielten sich die Bewohner des NFD lange Zeit relativ passiv und wurden von den Ereignissen im übrigen Kenia kaum berührt. An dem aufstrebenden afrikanischen Nationalismus und dem Mau-Mau-Aufstand nahmen sie keinen Anteil22. Erst im Zusammenhang mit dem sich nach dem Zweiten Weltkrieg in Italienisch-Somalia und Britisch-Somaliland entfaltenden Somali-Nationalismus und den Vorbereitungen auf die Unabhängigkeit Kenias um 1960 kam es im NFD zu

18 Lewis, Race (Anmerkung 14), S. 53.

$19 \mathrm{Zu} \mathrm{dem} \mathrm{"Zentrum-Peripherie"-Modell} \mathrm{vgl.} \mathrm{Senghaas,} \mathrm{Dieter} \mathrm{(Hrg.),} \mathrm{Imperialismus} \mathrm{und} \mathrm{strukturelle} \mathrm{Ge-}$ walt. Analysen über abhängige Reproduktion, Frankfurt/Main 1972.

20 Lewis Modern History, S. 184 und Race, S. 56 (Anmerkung 14).

21 Soja, E. W., The Geography of Modernization in Kenya, Syracuse, N. Y., 1968, S. 112.

22 Dazu vgl. vor allem Rosberg, Carl G. und Nottingham, John, The Myth of Mau Mau: Nationalism in Kenya, Nairobi 1966. 
einem regen politischen Leben ${ }^{23}$. Zwischen 1893 und 1918 hatten die Somali zwar bewaffneten Widerstand gegen die britische Kolonial-Administration geleistet, jedoch war dieser Widerstand politisch diffus geblieben und infolge von Kämpfen zwischen den Somali-Stämmen nicht umfassend organisiert und koordiniert gewesen. In den Jahren zwischen 1915 und 1943 kam es unter den in Nairobi lebenden wenigen Somali-Händlern zu begrenzten politischen Aktivitäten, die das Ziel hatten, für diese Somali den Status der Asiaten in Kenia zu erringen. Erst die Aktivitäten der „Somali Youth League“ (SYL), die 1943 in Mogadischu gegründet worden war, führten im NFD seit 1947 zu einer ersten politischen Massenbewegung. Binnen weniger Monate gewann die SYL und der von ihr propagierte Somali-Nationalismus unter den Somali, aber auch unter nicht-somalischen Bevölkerungsgruppen, eine große Anhängerschaft. Diese SYL-Aktivitäten führten zu einer ersten Artikulierung der sozialen und politischen Interessen der Bevölkerung des NFD und $\mathrm{zu}$ einer ersten umfassenden Organisierung politisch bewußt gewordener Gruppen. Da die Bewegung jedoch zunehmend einen anti-kolonialen Charakter annahm und die Autorität der britischen Verwaltung im NFD zu untergraben drohte, wurde die SYL Ende 1948 verboten. Von 1948 bis Ende der fünfziger Jahre gärte es im NFD wohl unterschwellig weiter, jedoch kam es erst im Zusammenhang mit der Unabhängigkeit der Republik Somalia und den Vorbereitungen auf die Unabhängigkeit Kenias im Jahre 1960 wieder zu einem Aufleben politischer Aktivitäten im NFD. Im Hinblick auf die im Jahre 1961 anstehenden Wahlen in Kenia ließen die Briten wieder parteipolitische Betätigung im NFD zu, und es bildeten sich dort eine ganze Anzahl von politischen Parteien. Die wichtigste dieser Parteien war zweifellos die "Northern Province People's Progressive Party“ (NPPPP), die offen eine Sezession des NFD von Kenia und einen Anschluß an die Republik Somalia forderte, und die bei der Mobilisierung der Massen und Rekrutierung von Mitgliedern die Erfolge der SYL von 1947/48 noch weit übertraf. Die überwiegende Mehrheit der Somali im NFD, aber auch andere Bevölkerungsgruppen, wünschten im Jahre 1960 offensichtlich den Anschluß an Somalia; sie verglichen ihren marginalisierten Minoritäten-Status im NFD mit den weitaus fortschrittlicheren Entwicklungen bei den in der Republik Somalia lebenden Somali und kamen zu dem Schluß, daß sie in Somalia ein besseres Leben führen könnten als in Kenia. Dieses Bewußtsein einer relativen Depravation ${ }^{24}$ hat wohl zusammen mit den ethnisch-kulturellen Identifikationen der Somali die wichtigste Rolle bei dem Wunsch nach Sezession im NFD gespielt.

Um nicht den Eindruck zu erwecken, als seien sie zu einer weiteren politischen Mitarbeit in Kenia bereit, boykottierten die Somali die Wahlen von 1961. In den Verhandlungen um die Unabhängigkeit Kenias ${ }^{25}$ vertraten die wichtigsten Parteien im NFD weiterhin den Standpunkt der Sezession und formulierten diesen Standpunkt im März 1962 in einem gemeinsamen Memorandum ${ }^{26}$. Die großen Parteien im Süden Kenias jedoch, die „Kenya African National Union“ (KANU) und „Kenya African Democratic Union“ (KADU), die die überwältigende Mehrheit der Bevölkerung Kenias repräsentierten, lehnten eine Sezession des NFD kategorisch ab. Offensichtlich um Zeit zu gewinnen und diese kontroversen Standpunkte etwas aufzulockern, ließ die britische Regierung die Meinung der NFD-

23 Zum Folgenden vgl. vor allem Turton (Anmerkung 14).

24 Vgl. dazu Gurr, Ted, Why Men Rebel, Princeton 1970, S. 329

$25 \mathrm{Vgl}$. Report of the Kenya Constitutional Conference, Cmnd 1700

26 A. People in Isolation: A Call by Political Parties of the NFD of Kenya for Union with the Somali Republic, London, März 1962. 
Bevölkerung durch eine unabhängige Kommission testen. Die Ergebnisse der Kommission ließen keinen $Z$ weifel daran, daß eine klare Mehrheit der Bevölkerung des NFD die Sezession mit dem Ziel eines Anschlusses an die Republik Somalia wünschte ${ }^{27}$. Die überwältigende Mehrheit aller Somali, aber auch große Teile der nicht-somalischen Moslem-Bevölkerung des NFD sprachen sich für die Sezession aus. Die Grenze von Pro und Contra war etwa deckungsgleich mit der Grenze zwischen Moslems und Nicht-Moslems. Doch ohne auf diese Ergebnisse und das Drängen der Republik Somalia auf Selbstbestimmung für die Bevölkerung des NFD Rücksicht zu nehmen, löste Großbritannien im Frühjahr 1963 die administrativen Grenzen des alten NFD auf, konstituierte aus den alten Sub-Distrikten neue Regionen ${ }^{23}$ und entließ die fast ausschließlich von Somali bewohnten östlichen Teile des alten NFD als neue „North-Eastern Region“ (NER) und integralen Bestandteil Kenias Ende 1963 mit in die Unabhängigkeit. Diese Entscheidung Großbritanniens dürfte wohl nicht zuletzt unter dem Druck der KANU und der äthiopischen Regierung getroffen worden sein; in jedem Fall aber waren auch die britischen (strategischen, ökonomischen und politischen) Interessen in Kenia vergleichsweise bedeutender als die in der Republik Somalia ${ }^{29}$. Folge dieser Entscheidung waren im NFD zunächst sporadische Unruhen und vereinzelte Zwischenfälle, dann, im November 1963, also noch vor der endgültigen Unabhängigkeit Kenias, der Ausbruch des shifta-Krieges.

\section{Guerilla und Counter-Guerilla ${ }^{30}$}

Die Charakterisierung des shifta-Krieges als eines Krieges, der „half guerilla, half cattle-rustling" war, stellt zwar eine grobe Vereinfachung dar, enthält jedoch auch einen Kern Wahrheit ${ }^{31}$. Versucht man das Gewaltmuster der shifta-Guerilla in seine Bestandteile $\mathrm{zu}$ zerlegen, so trifft man auf eine eigenartige Mischung von traditionalen und modernen Elementen. Kriegerische Traditionen des ursprünglichen Widerstandes der Somali-Nomaden gegen die britische Kolonialherrschaft ${ }^{32}$ dürften ebenso eine Rolle gespielt haben wie Elemente traditionaler Stammeskriege, also etwa das, was Colin Leys in seiner Typologie von Gewalt in Afrika als "war subsystems" bezeichnet hat ${ }^{33}$. Van Berghe wies darauf hin, daß die shifta-Einheiten noch vielfach dem Typus einer traditionalen „Raiding Citizen Army“ segmentärer Gesellschaften entsprachen ${ }^{34}$. Andererseits jedoch finden wir bei der shifta-Guerilla in zunehmendem Maße auch Elemente des modernen neuzeitlichen Kleinkrieges vor ${ }^{35}$.

Die shifta-Guerilla war zunächst wahrscheinlich nicht, wie vielfach behauptet wurde, von der Republik Somalia aus initiiert und ferngesteuert, sondern eine weitgehend spontane und authentische Bewegung der Bevölkerung im NFD, wenn-

27 Vgl. Report of the Northern Frontier District Commission, Cmnd 1900.

28 Nach den Empfehlungen des Report of the Regional Boundaries Commission Cmnd 1899.

29 Zur britiscinen Position vgl. Drysdale (Anmerkung 14).

30 Dieser Abschnitt gründet sich vor allem auf die Kenia-Dokumentation „Kenya-Somalia-Relations“, Nairobi 1967, und auf das Africa Researds Bulletin.

31 Lewis, Roy, Times, 30. März 1967.

32 Vgl. Turton (Anmerkung 14), S. 142.

33 Leys, Colin, Types of Violence, in: Transition, vol. 5 , No, 21, S. 17-20.

34 Van den Berghe, Pierre L., The Military and Political Change in Africa, in: Welch C. E. Jr. (Ed), Soldier and State in Africa, Evanston 1970, S. $254 \mathrm{f}$.

35 Vgl. dazu z. B. Freiherr von der Heydte, Friedrich A., Der Moderne Kleinkrieg als wehrpolitisches und militärisches Phänomen, Würzburg 1972; Hahlweg, Werner, Typologie des Modernen Kleinkrieges, Münster 1967; Tinker, Jerry $M$. et alia (Eds), Strategies of Revolutionary Warfare, Neu Delhi 1969; Paret, Peter und Shy, John W., Guerilias in the 1960's, N. Y. 1962. 
gleich sie im Verlauf des Krieges auch in immer stärkerem Maße von der materiellen, finanziellen und organisatorischen Unterstützung durch die Republik Somalia abhängig wurde. Am besten könnte man die shifta-Guerilla vielleicht, besonders in ihrer Anfangsphase, als eine „autonom-kooperierende“ Bewegung bezeichnen ${ }^{37}$. Ihre soziale Basis war die gesamte pro-sezessionistische Bevölkerung des NFD, besonders aber die Somali-Stämme entlang der Grenze zu Somalia. Nachdem die sezessionistische Bewegung im NFD politisch keinen Erfolg gehabt hatte, griff sie nun zum Mittel der Gewalt um die Politik der Kenia-Regierung gegenüber der NFD-Bevölkerung zu ändern ${ }^{38}$. Die aktiven Kämpfer der shifta-Bewegung dürften sich überwiegend aus den jungen Männern der NFD-Somali rekrutiert haben; „Freiwillige“ aus der Republik Somalia spielten nur eine untergeordnete Rolle. Die Intensität der Sympathie und der Grad der aktiven Unterstützung für die shifta-Guerilla in der Bevölkerung variierten allerdings regional und zeitlich; sie dürften vor allem abhängig gewesen sein von der jeweiligen Distanz zur SomaliaGrenze bzw. zu den Kontrollzentren der Regierung. Im Laufe des Krieges aber scheinen sich Teile der Bevölkerung zum Schutze ihrer unmittelbaren Lebensinteressen unter dem zunehmenden Druck der Regierungstruppen und unter dem Eindruck eines wachsenden Terrors der shifta gegen die Zivilbevölkerung auf eine Position der passiven Neutralität bzw. der Indifferenz gegenüber den Kriegsparteien zurückgezogen zu haben. Ein kleinerer Teil der NFD-Bevölkerung, der von Beginn an loyal geblieben war, ging sogar zu einer aktiven Kollaboration mit den Regierungstruppen über.

Die regionale Basis der shifta konzentrierte sich vor allem auf die Distrikte Garissa, Wajir und Mandera entlang der Grenze zu Somalia. Doch breitete sich die Guerilla regional auch weiter aus zur Nordküste im Lamu-Distrikt, entlang dem Tana-Fluß in Teile des Kitui- und Meru-Distrikts und sogar bis in Teile des Rift Valley am Rande der dichter besiedelten Gebiete Kenias ${ }^{39}$. Zeitweilig hatte es sogar den Anschein, als könne sich eine Allianz zwischen den shifta und den Guerillas der „Kenya Land Freedom Army “ (ehemaligen Mau-Mau-Kämpfern) bilden, die ihre Basis in den Wäldern nördlich des Mount Kenya hatten ${ }^{40}$. Doch schwand diese Gefahr für Kenia in dem Maße, wie sich die alten Mau-Mau-Kämpfer ergaben. Verschiedene Angriffe wurden bis in die Stadt Isiolo hinein vorgetragen, doch gelang es den shifta nie, den Krieg auf das Kernland Kenias auszudehnen. Die Zahl der aktiven shifta-Guerillas gab Premierminister Kenyatta im Dezember 1963 für die NER mit etwa 700 an, nicht eingerechnet etwa 2000 weitere shifta, die in Lagern in Somalia ausgebildet wurden ${ }^{41}$. Verteidigungsminister Mungai schätzte die Zahl der shifta im Herbst 1964 auf etwa 800 bis 100042. Doch muß die Zahl der aktiv kämpfenden shifta schon zu dieser Zeit weitaus höher gewesen sein, denn bereits im August 1964 wurde von shifta-Gruppen in Stärke von 200 und 300 Mann berichtet, die den kenianischen Sicherheitsstreitkräften Gefechte lieferten ${ }^{43}$.

\footnotetext{
36 Vgl. dazu den Somali Yousuf Jama Ali Duhul, der diese Annahme als "universal misconception“ bezeichnet, in: Doob, Leonard W. (Ed), Resolving Conflict in Africa, New Haven und London 1970, S. 49.

37 Begriff nach Schwarz, Hans-Peter, unveröffentlichtes Arbeitspapier über Internationale Gewaltorganisationen, Hamburg, Herbst 1972.

$38 \mathrm{Vgl}$. dazu die weite Definition von „internal war“ durch Eckstein (Anmerkung 7), S. 1: „Broadly speaking, internal wars are attempts to change by violence, or threat of violence, a government's policies, rulers, or organization."

39 Vgl. Soja (Anmerkung 21), S. 113

40 Vgl. Soja (Anmerkung 21), S. 113 (Anmerkung 17).

41 Kenya-Somalia-Relations, Nairobi 1967 , S. 15.

42 Vgl. Republic of Kenya, Official Report, House of Representatives, First Parliament, Second Session, vol. 3, Part II, 8. Sept. 1964, col. 2101.

43 Vgl. Kenya-Somalia-Relations, Nairobi 1967, S. 27.
} 
Die Bewaffnung der shifta bestand überwiegend aus älteren italienischen und britischen Gewehren; doch tauchten im Verlauf des Krieges auch zunehmend moderne Schnellfeuerwaffen wie z. B. Maschinenpistolen auf, später auch Sprengstoffe und Landminen.

Über die Organisation der shifta ist relativ wenig bekannt ${ }^{44}$. Offensichtlich saß ihre Kommando-Zentrale, das „High Command“ der „Northern Frontier District Liberation Movement“ im Exil in Mogadischu, während für die Kampfzonen in Kenia, die sich weitgehend mit den Distrikten deckten, einzelne militärische Führer verantwortlich waren. Insgesamt scheint die shifta-Organisation infolge schlechter Ausbildung, mangelnder Disziplin und ungenügender Führung sehr instabil und ineffektiv gewesen zu sein. Das strategische Konzept der shifta, wenn man überhaupt von einem zusammenhängenden strategischen Konzept sprechen kann, bestand wohl darin, die gesamte Verwaltung des NFD lahmzulegen und die zivilen und militärischen Vertreter der Regierung aus dem Distrikt zu verdrängen; ob die shifta darüber hinaus planten, die dichter besiedelten Zentren Kenias und den Tourismus zu beunruhigen, ist umstritten ${ }^{45}$. Die Taktiken und Techniken, die die shifta anwandten, reichten von Attentaten, Menschenraub, Sabotage, Überfällen bis hin zu systematischem Terror ${ }^{46}$ und kleineren offenen Gefechten. Geht man bei der Beurteilung der shifta-Strategie von der seit Mao Tse-tung berühmten Gliederung des Guerilla-Krieges in drei Eskalationsphasen aus ${ }^{47}$, so erreichten die shifta sicherlich die erste, bestenfalls die zweite, jedoch kaum die dritte Phase.

Der Krieg begann im November 1963 und setzte sich dann im Jahre 1964 fort mit Überfällen auf Polizeiposten, Armeelager, dukas (Krämerladen) und manyattas (nomadische Siedlungen mit Umhegungen für das Vieh), mit Attentaten auf loyale Somali- und Kenia-Beamte sowie Entführungen von loyalen Somali-Politikern. Unter den Opfern dieser Aktionen waren auch verschiedene Europäer. Im Jahre 1965 scheint die shifta-Guerilla infolge von organisatorischer Desintegration, Versorgungsschwierigkeiten und wachsenden Erfolgen der Regierungstruppen eine Schwächeperiode durchgemacht $\mathrm{zu}$ haben. Im Jahre 1966 intensivierte sich der Krieg wieder, insbesondere durch die erstmalige Verwendung von Sprengstoffen und Landminen ${ }^{48}$. Durch den massiven Einsatz von Landminen im Jahre 1967, bei dem die Straßen im NFD vermint wurden, fügten die shifta den Regierungstruppen, aber auch zivilen Reisenden schwere Verluste zu. Der Verkehr im NFD kam fast völlig zum Erliegen. Doch führte diese Verschärfung des Krieges auch zu energischen Protesten Kenias gegen die militärische Unterstützung der shifta durch die Republik Somalia. Im Herbst 1967 kam es dann aus Gründen, die uns hier nicht näher zu beschäftigen brauchen, zu Verhandlungen zwischen Kenia und Somalia, die einen Stopp der somalischen Intervention zur Folge hatten und zusammen mit gezielten Counter-Guerilla-Maßnahmen der Kenia-Regierung zu einem weitgehenden Abflauen der Kampfhandlungen führten ${ }^{49}$.

44 Vgl. dazu Kenya-Somalia-Relations, Nairobi 1967, S. 54, 60.

45 Vgl. dazu. Kenya-Somalia-Relations, S. 318. Vermutungen in dieser Richtung basieren auf einem Artikel von Dennis Neeld in der kenianischen Zeitung „Daily Nation“ im November 1966 über einen "Secret Shifta-Plan to attack Kenya".

$46 \mathrm{Vgl}$. dazu Thornton, Thomas P., Terror as a Weapon of Political Agitation in: Eckstein (Anmerkung 7), S. 71-99.

47 Vgl. dazu Mao Tse-tung, Theorie des Guerilla-Krieges oder Strategie der Dritten Welt, Reinbek bei Hamburg 1266; vgl. auch Suggs, Robert C., und Wolak, Brenda M., Phases of Development, in: Tinker, Jerry M. et alia (Anmerkung 35ं), S. 43-54."

48 Vgl. Kenya-Somalia-Relations, S. 46, 48.

49 Einen knappen Ǔberblick über diese Verhandlungen gibt Howell, John, An Analysis of Kenyan Foreign Policy, in: Journal of Modern African Studies, vol. 6, No. 1, 1968, S. 38 ff. 
Die Counter-Guerilla der Regierung umfaßte sowohl militärische als auch administrativ-politische Maßnahmen einschließlich der einschlägigen Techniken von Civic Action, Verbotenen Zonen etc. ${ }^{50}$. Auf dem militärischen Gebiet konnten die Sicherheitsstreitkräfte Kenias ${ }^{51}$, die von Großbritannien stark unterstützt wurden ${ }^{52}$, auf die Erfahrung der Briten im Mau-Mau-Aufstand und in Malaya zurückgrei$f{ }^{53}$. Zunächst lag die Bekämpfung der shifta vor allem in den Händen der Polizei, bei der sich insbesondere die GSU (= „General Service Unit“, eine Art von para-militärischer Bereitschaftspolizei) und der "Air Wing“ hervortaten, dessen Piloten meist Engländer waren, die schon im Mau-Mau-Aufstand mitgekämpft hatten ${ }^{54}$. Später jedoch kamen auch immer mehr Einheiten der "Kenya-Rifles“ zum Einsatz ${ }^{55}$. Die Regierungstruppen operierten meist in kleineren Einheiten; gelegentlich jedoch kam es auch zu größeren Operationen wie z. B. im August 1966, als von einer Basis im Lamu-Distrikt aus Einheiten der Army, Air Force, GSU und Navy die Operation „Shambulia Sana“ (= „Operation All Out Attack“) starteten ${ }^{56}$. Die bessere Ausbildung, Ausrüstung und Bewaffnung (Fahrzeuge, Funkgeräte, Logistik etc.) verlieh den Regierungstruppen im offenen Kampf Überlegenheit; doch wurde diese Überlegenheit weitgehend wieder ausgeglichen durch die bessere Ortskenntnis, Beweglichkeit und Ausdauer der shifta.

Die administrativ-politischen Maßnahmen der Regierung sollten die militärischen Maßnahmen erleichtern und flankieren. Seit der Unabhängigkeit im Dezember 1963 besaß die Regierung weitgehende Ausnahmerechte für die NER, die später auf die Distrikte Marsabit, Isiolo, Tana River und Lamu ausgedehnt wurden ${ }^{57}$. Am 1. September 1964 wurden spezielle Sicherheitsmaßnahmen für die NER und Teile der Eastern und Coast Region ergriffen ${ }^{58}$. Am 8. Juli 1966 ergriff die Regierung weitere einschneidende Maßnahmen: eine Registrationspflicht für alle Somali und eine Zwangsansiedlung der Somali und anderer Bevölkerungsgruppen in manyattas unter dem Schutz und der Kontrolle der Behörden und Sicherheitsstreitkräfte ${ }^{59}$. Im Sommer 1967 wurde die Registrationspflicht sogar auf diejenigen Somali ausgedehnt, die in der Central Province, in der Nairobi Area und im Mombasa Distrikt lebten ${ }^{60}$. Alle diese Maßnahmen, insbesondere aber die Zwangsansiedlung in manyattas, verfolgten unzweifelhaft das Ziel, den shifta-Guerillas das "Wasser“, in dem sie nach einem poetischen Bild Mao Tse-tungs „wie die Fische schwammen“, abzugraben, ihnen also die Unterstützung der Bevölkerung zu entziehen ${ }^{61}$. Längerfristig gesehen verfolgte die Regierung mit diesen Maßnahmen wohl ganz allgemein das

50 Vgl. dazu z. B. McCuen, John J., The Art of Counter-Revolutionary War, London 1966; Pustay, John S., Counterinzsurgency Warfare, N.' Y., London 1965; Galula, David, Counter-Insurgency Warfare, London und Dunmow 1964.

51 Vgl. den Ưberblick bei Kaplan, Irving et alia, Area Handbook for Kenya, Washington, D. C., Juli 1967, über die Polizei und Armee in Kenva.

52 Zur Militärhilfe Großbritanniens an Kenia vgl. SIPRI, The Arms Trade with the Third World, Stockholm 1971, S. 639-642.

53 Zum Aufstand in Malaya siehe Horlemann, Jürgen, Modelle der kolonialen Konterrevolution, Frankfurt/Main 1968, S. 7-73.

54 Vgl. Kaplan (Anmerkung 51), S. 631.

55 Einen Eindruck vom Einsaiz der Aruee vermittelt Gertzel, Cherry, Goldschmidt, Maure und Rothchild Donald (Eds), Government and Politics in Kenya, Nairobi 1969, S. 572-573: „The Army in Action: the War Against the Shift".a

56 Africa Research Bulletin, 1966, 593 B.

57 Vgl. Ghai, Y. P., Independence and Safeguards in Kenya, in: East African Law Journal, 3, 1963, S. 183.

58 Kenya Gazette Supplement No. 115 (Legislative Supplement No. 43), 1. Sept. 1964 (The Public SecurityNorth Eastern Region and Contiguous Districts-Regulations, 1964):

$59 \mathrm{Vgl}$. Africa Research Bulletin, 1966, 5\%5 B; vgl. auch Kenya-Somalia-Relations, S. $48 / 49$.

60 Vgl. Africa Research Bulletin, 1967, 821 BC.

61 Zur Ansiedlingskampagne vgl. Deutsche- Welle-Dokumentation (Anton Fendrich, Reiseeindrücke aus der Nordostprovinz), 6. 9. 1967, S. 3, 5, 6, 8; Handelsblatt (Düsseldorf) vom 6. 9. 1967; Kenya-SomaliaRelations, S. 49; Legum, Colin Kenya's Little Guerilla War Heats Up, in: Africa Report, April 1967, S. 39; nach Fendrich, S. 5, gab der kenianische Verteidigungsminister Mungai vor der Presse an, daß von den etwa $220000 \mathrm{im}$ shifta-Gebiet lebenden Menschen schon etwa $80 \%$ in manyattas angesiedelt seien. 
Ziel, die Nomadenbevölkerung des alten NFD seßhaft zu machen; zum einen, um sie dadurch politisch-administrativ und militärisch besser kontrollieren zu können, zum anderen aber wohl auch, um durch zunehmende Urbanisierung die Grundlagen für eine ökonomische und soziale Entwicklung zu schaffen, die den Lebensstandard der NFD-Bevölkerung heben und sie enger an den kenianischen Staat binden sollte. Hinsichtlich der Kosten des shifta-Krieges seien hier nur die relativ gut meßbaren physischen und finanziellen Kosten genannt; auf die allgemeinen sozio-ökonomischen Kosten für den NFD, dessen Weidewirtschaft weitgehend beeinträchtigt wurde, und die allgemeinen menschlich-sozialen Kosten für seine Bevölkerung (Gefangenschaft, Krankheit, Hunger, Zerstörung von Sozialstrukturen etc.) sei hier nur hingewiesen. Die finanziellen Kosten für die Kriegführung im NFD gab Verteidigungsminister Mungai auf einer Presse-Konferenz am 21. August 1967 mit drei Mill. £ pro Jahr an ${ }^{62}$. John Drysdale, ein guter Kenner der Szenerie, schätzte die Kosten allein für die Jahre $1966 / 67$ auf „some 10 Mill. £63. Die Zahl der Toten des Krieges, darunter meist shifta und somalische Ziviltote, gab Präsident Kenyatta am 15. Februar 1967 mit etwa 2000 an $^{64}$. Nach Schätzungen von Howell und Potholm aber beläuft sich die Zahl der Gesamttoten des shifta-Krieges auf 4000 bis $6000^{65}$.

\section{Die Position des Status quo: Legitimitätsprobleme und interne Domino-Theorie}

Die Ursachen dafür, warum die Somali in Kenia zum Mittel der Gewalt griffen und einen Guerilla-Krieg begannen, haben wir bereits erörtert. Aus welchen Gründen aber lehnten die großen Parteien KANU und KADU und die kenianische Regierung eine Sezession im NFD ab und reagierten auf die shifta-Guerilla mit einer energischen Counter-Guerilla? War es nur nationaler Stolz ${ }^{66}$ oder die vage Aussicht auf Ölfunde im NFD ${ }^{67}$ ? Sicherlich ist kaum ein Staat bereit, freiwillig große Teile seines Territoriums abzutreten, besonders dann nicht, wenn der ökonomische Wert der umstrittenen Gebiete noch steigen könnte. Dennoch scheinen uns dies mehr oder weniger oberflächliche Antworten auf die Frage zu sein, warum die Kenia-Regierung die hohen Kosten des Krieges auf sich nahm, um ein peripheres, relativ dünn besiedeltes, arides und ökonomisch bisher weitgehend wertloses Steppengebiet als integralen Bestandteil Kenias zu erhalten. Es ist zu vermuten, daß im Kalkül der Kenia-Regierung die potentiellen Kosten einer erfolgreichen Sezession im NFD noch weitaus höher eingeschätzt wurden als die tatsächlichen Kriegskosten, und in diesem Kalkül finden wir den Schlüssel zur Beantwortung unserer obigen Frage.

Betrachten wir die Argumentation der führenden Politiker der KANU und KADU sowie der Kenia-Regierung zugunsten ihrer Position des status quo im NFD, so treffen wir immer wieder zwei zentrale und eng miteinander verbundene Begründungen dieser Position an ${ }^{63}$. Die eine Begründung beruht auf der These, daß eine

62 East African Standard, 22. August 1967.

63 Drysdale, John, The Situation in December 1967 - Review and Prospect, in: Hoskyns (Anmerkung 4), S. 84 .

64 Republic of Kenya, The National Assembly, Official Report, First Parliament, Fifth Session, vol. XI, 15, Februar 1967, col. 14.

65 Howell (Anmerkung 49), S. 39; Potholm, Christian P., Four African political systems, Englewood Cliffs, N. J., 1970, S. 225.

66 So z. B. Lew is, Race (Anmerkung 14), S. 60.

67 Zur Olsuche im NFD vgl. Times, 18. 11. 1963; auf die Frage eines Journalisten hinsichtlich der Bedeutung von Olfunden im NFD soll Verteidigungsminister. Mungai geantwortet haben, daß, falls Öl gefunden werde, die Somali dieses sicher ebenso gern besitzen würden wie die Kenianer, vgl. DeutscheWelle-Dokumentation (Anmerkung 61), S. 8.

$68 \mathrm{Vgl}$. dazu vor allem das von der Kenia-Delegation zur OAU-Gründungskonferenz von 1963 in Addis Abeba vorgelegte Memorandum "Pan-African Unity and the NFD Question in Kenya", hier zitiert in Hoskyns (inmerkung 4), S. 36-39 und die schon mehrfach zitierte Kenia-Dokumentation "Kenya-SomaliaRelations“, Nairobi 1967. 
Sezession der Somali ähnliche Bestrebungen bei anderen Bevölkerungsgruppen Kenias auslösen und damit einen territorialen Desintegrationsprozeß in Gang setzen würde, der letztlich eine Auflösung des Staates zur Folge hätte. Diese These könnte man als interne Domino-Theorie bezeichnen. Es sei hier noch erwähnt, daß diese These in Kenia zu einer Zeit formuliert wurde, als den Kenia-Politikern das abschreckende Beispiel des Kongo und der Katanga-Sezession vor Augen stand; diese Kongo-Erfahrungen spielten bei der damaligen Definition der politischen Situation durch die Kenia-Politiker sicher eine gewisse Rolle ${ }^{69}$. Die andere Begründung läßt sich als ein Bekenntnis zum Prinzip der Staatensukzession kennzeichnen. Sie geht von der Prämisse aus, daß die jungen afrikanischen Staaten an den kolonial vererbten Grenzen und Territorien festhalten und innerhalb dieser Grenzen und Territorien die Prozesse der Nationenbildung betreiben müssen. Die Konstituierung von Staaten auf der Basis von ethnisch-kulturellen Identitäten, wie sie etwa die Somali anstrebten, sei abzulehnen, da sie bei der mangelnden ethnisch-kulturellen Kohäsion der meisten afrikanischen Staaten zu einer anarchischen Balkanisierung Afrikas führen würde. Das von den Somali geforderte Recht auf Selbstbestimmung gelte daher nur "where foreign domination is the issue. It has no relevance where the issue is territorial disintegration by dissident citizens "70.

Welche realsoziologischen Phänomene lagen diesen beiden theoretischen Begründungen des Status quo zugrunde? Als ein typischer neuer Staat der Dritten Welt hatte Kenia das große Problem der Nationenbildung zu lösen ${ }^{71}$. Die Bevölkerung Kenias war ethnisch-kulturell heterogen zusammengesetzt; soziale, ökonomische und politische Bedingungen verschärften noch die Unterschiede zwischen den einzelnen Gruppen der Bevölkerung, die miteinander um politische Macht, Einfluß und die Verteilung von knappen Ressourcen konkurrierten ${ }^{72}$. Aufgrund verschiedener Faktoren war es insbesondere der größten ethnischen Gruppe Kenias, den Kikuyu, gelungen, eine dominante politische Position einzunehmen. Der kenianische Staat gründete sich nicht auf eine die Bevölkerung einigende nationale Identifikation, sondern in erster Linie auf die koloniale Tradition gemeinsamer Grenzen und Verwaltung und den Machtanspruch der Kikuyu. Kenia war als Staat primär definiert durch die kolonial vererbten Grenzen und territorialen Besitzstände; seine Legitimitätsquelle war überwiegend diese koloniale territoriale Traditon ${ }^{73}$. Erst in zweiter Linie bezog der Staat seine Legitimität von einem „territorialen Nationalismus"74, der versuchte, parochiale Loyalitäten und Identifikationen umzuorientieren und auf die zentralen administrativ-politischen Strukturen $z u$ projizieren ${ }^{75}$. Kenia teilte mit anderen jungen Staaten das Problem mangelnder horizontaler und vertikaler Integration ${ }^{76}$. Zwischen ethnisch-territorial

$69 \mathrm{Vgl}$. z. B. d:e Außerung Kenyattas im Jahre 1961: „Four or Part, looking at the problems of the Congo, we do not want another Congo in Kenya! «, zitiert in: Africa Confidential, No. 24, 1961, S. 3.

70 Pan-African Unity and the NFD Question in Kenya (Anmerkung 68), S. 39.

$71 \mathrm{Vgl}$. dazu allgemein Nuscheler, Franz, Theorien zur politischen Entwicklung, in: CIVITAS, Bd. 8, 1969, S. 73-79; Deutsch, Karl W., Der Nationalismus und seine Alternativen, München 1972, bes. 69-93; Tibi, Bassam, Nationalismus in der Dritten Welt am arabischen Beispiel, Frankfurt/Main 1971.

72 Zur Bevölkerungsstruktur Kenias vgl. Morrison, D. G. et alia, Black Africa. A Comparative Handbook, N. Y., 1972, S. 270-276; Internationales Afrika-Forum, Jg. 5, H. 12, 1969, S. 755-759 (= Studie zum Tribalismus in Kenia); einen ausgezeichneten Ưberblick einschließlich der politischen Implikationen geben Gertzel, Goldschmidt, Rothchild (Anmerkung 55) und Gertzel, Cherry, The Politics of Independent Kenya 1963-68, Nairobi-London 1970.

73 Vgl. Touval, Saadia, The Sources of Status quo and irredentist policies, in: Widstrand (Ed) (Anmerkung 6), S. 104-108; ders., The Shifta Warfare, in: East Africa Journal, Mai 1966, S. 7-10.

74 Zum Begriff vgl. Smith, A. D., Theories of Nationalism, London 1971, S. 217.
75 Vgl. dazu Berg-Schlosser, Dirk, Die Problematik des "nation-building ${ }^{*}$ in Afrika aufgezeigt am Beispiel Kenias, in Internationales Afrika Forum, 4. Jg., H. 7/8, Juli/August 1968, S. 457-462.

76 Vgl. dazu Fröhlich, Dieter, Multilingualismus und der Aufbau der Nation, in: König, René (Hrg.), Aspekte der Entricklungssoziologie, Köln und Opladen 1969, S. 473. 
definierbaren Teileinheiten seiner Bevölkerung gab es bestenfalls erst rudimentäre gemeinsame politische Loyalitäten, wie es innerhalb seiner Bevölkerung auch noch erhebliche sozio-ökonomische Ungleichgewichtigkeiten gab. Da die Kikuyu als ethnisch-territorial definierbare Gruppe die anderen Bevölkerungsgruppen sowohl politisch als auch sozial und ökonomisch dominierten, hatten sich in Kenia die Probleme mangelnder horizontaler und vertikaler Integration auf gefährliche Weise miteinander verbunden. Diese Situation führte $\mathrm{zu}$ vielfältigen Bedrohungen der politischen Stabilität ${ }^{77}$ : Es sei hier nur hingewiesen auf die Sezessionstendenzen bei den Arabern im Küstenbereich ${ }^{78}$ und den Masai ${ }^{79}$, auf die latenten Spannungen zwischen der von Kikuyu und Luo getragenen KANU und der KADU, die eine Allianz des Mißtrauens ethnischer Minoritäten gegenüber der Kikuyu-Luo Allianz darstellte, sowie auf die Fragilität dieser Kikuyu-Luo-Allianz selbst. Die schon erwähnte Rebellion der „Kenya Land Freedom Army“ und inter-ethnische Kämpfe im Turkana-Gebiet waren neben der shifta-Guerilla die wichtigsten Manifestationen „kommunalistischer" Instabilität in Kenia ${ }^{80}$. Die Bedrohungen, die sich aus dieser instabilen politischen Situation für Kenia ergaben, waren im wesentlichen von zweierlei Art: Zum einen wurde die territoriale Integrität des Staates bedroht, zum anderen die Legitimität seiner zentralen politisch-administrativen Instanzen ${ }^{81}$.

Betrachten wir nun den shifta-Krieg im Kontext dieser allgemeinen politischen Instabilität in Kenia, so war die shifta-Guerilla eindeutig ein „irredentistischer“ Typ von Instabilität ${ }^{82}$, der die territoriale Integrität des Staates bedrohte. Der NFD lag in einer Zone kompetitiver nationaler Identifikation und sozialer Mobilisierung ${ }^{83}$; der ethnisch-kulturelle Nationalismus der Somali und der sich auf die kolonialen Grenzen gründende territoriale Nationalismus der Kenia-Regierung waren inkompatibel. Doch war die interne Domino-Theorie wirklich valide ${ }^{84}$ ? In gewisser Weise stellten die Somali innerhalb Kenias einen Sonderfall dar ${ }^{85}$; keine andere sezessionsverdächtige Bevölkerungsgruppe war politisch so gut organisiert und lebte geschlossen an der Grenze zu einem Staat, dessen Bevölkerung und Regierung die Sezessionstendenzen so entschlossen unterstützten. Eine erfolgreiche Sezession der Somali hätte daher kaum eine sezessionistische Kettenreaktion und damit die territoriale Desintegration Kenias ausgelöst. In einem mechanistischen und automatistischen Sinne war die interne Domino-Theorie daher nicht valide. Wie wir aber gesehen haben, beruhte die Legitimität des Staates und seiner Regierung vor allem auf dem territorialen Erbe der Kolonialzeit. Ein Nachgeben der Regierung in der Somali-Frage hätte daher ihre eigene Legitimität bedroht und wahrscheinlich zu einer allgemeinen staatlichen Legitimitäts- und Autoritätskrise geführt, da das Nachgeben der Regierung vermutlich von anderen unzufriedenen

$77 \mathrm{Vgl}$. dazu allgemein Morrison et alia (Anmerkung 72), S. $274 \mathrm{f}$.

$78 \mathrm{Vgl}$ dazu Salim, A. I., The Morement for "Mwambao “ or Coast Autonomy in Kenya, 1956-1963, in: Hadith 2, Nairobi 1970, S. 212-228; Ghai (Anmerkung 57), S. 183-187; Okumu, John., in: Doob (Ed), (Anmerkung 36), S. 62-64; Melamid, Alexander The Kenya Coastal Strip, in Geographical Review, vol. 53,1963 , S. $457-59$.

79 Vgl. dazu Ghai (Anmerkung 57), S. 187 f.; Okumu, John J., in: Doob (Ed), Anmerkung S. 61 f.

$80 \mathrm{Zum}$ Begriff politischer Instabilität in Afrika im Allgemeinen und kommunalistischer Instabilität im Besonderen vgl. Morrison et alia (Anmerkung 72), S. 122-131.

$81 \mathrm{Zu}$ dieser Unterscheidung vgl. Matthews, R. O., Domestic and Inter-State Conflict in Africa (Anmerkung 5), S. $460 \mathrm{f}$.

82 Vgl. Morrison et alia (Anmerkung 72), S. 129, wo er als "irredentism“ einen Vorgang bezeichnet, bei dem "an identifiable communal group seeks to change its political allegiance from the government of the territorial unit in which it resides to a political system, either existing or to be created, in which the authorities share the communal identification of the irredentist group concerned".

83 Vgl. Soja (Anmerkung 21), S. 113.

84 Zum Folgenden vgl. Howell (Anmerkung 49), S. $41 \mathrm{f}$.

$85 \mathrm{Vgl}$. Castagno (Anmerkung 14), S. 188. 
Bevölkerungsgruppen als eine politische Schwäche der zentralen staatlichen Instanzen ausgelegt worden wäre. Eine erfolgreiche Somali-Sezession hätte somit möglicherweise einen Demonstrations- und spill-over-Effekt gehabt und andere Bevölkerungsgruppen zum Ungehorsam gegenüber dem Staate und seiner Regierung ermuntert. Dadurch aber wären sowohl die ohnehin fragilen Grundlagen des Staates als auch möglicherweise die Dominanz der die Regierung tragenden Kikuyu erschüttert worden. Mit diesen Implikationen für die Innenpolitik Kenias war der shifta-Krieg dann nicht mehr nur ein „tribal war“, der mit seinen sezessionistischen Zielsetzungen die territoriale Integrität des Staates gefährdete, sondern gleichzeitig auch ein "anti-regime war" 86 oder "authority/structural war" 87 , der die Legitimität des Staates und seiner Regierung bedrohte und damit an Grundfragen der staatlichen Existenz Kenias rührte.

\section{Schlußbetrachtung und Ausblick}

Fassen wir abschließend die Grundzüge des shifta-Krieges noch einmal zusammen: Er war

- ein innerer Krieg im Sinne der weiten Definition von Eckstein ${ }^{88}$, ein authentischer innerer Krieg mit allerdings starken Interventionen auf beiden Seiten (KeniaGroßbritannien/Shifta-Somalia). Bezieht man diese externen Einflüsse in eine Analyse des shifta-Krieges ein, so könnte man in ihm Züge eines StellvertreterKrieges (Großbritannien contra Somalia bzw. Nachfolgestaat Kenia contra Somalia) erkennen, bei dem die Ziele von externem und internem Akteur jedoch kompatibel waren; am besten ließe sich der shifta-Krieg jedoch als ein „gemischter Krieg " im Sinne von Karl Deutsch bezeichnen ${ }^{89}$,

- von seiner Genesis her ein Krieg mit anti-kolonialen Zügen, in dem eine Bevölkerungsgruppe gegen Großbritannien bzw. dessen Nachfolge-Staat Kenia um ihre Unabhängigkeit kämpfte,

- auf der Problem-Ebene der vertikalen, sozialen Integration ein Krieg einer ethnisch-territorial definierbaren marginalisierten Peripherie gegen ein ebenfalls ethnisch-territorial definierbares Zentrum,

- auf der Problem-Ebene der horizontalen, territorialen Integration ein Krieg zwischen zwei Akteuren mit inkompatiblen nationalen Identifikationen, ein Krieg, der zwar primär die territoriale Integrität Kenias bedrohte, sekundär jedoch auch die Legitimität des Staates und seiner Regierung,

- im Sinne der konflikttheoretischen Annahmen der modernen Friedens- und Konfliktforschung von seiner Ausgangslage her ${ }^{90}$ ein asymmetrischer Konflikt zwischen einem "topdog“ (der Regierung Kenias) und einem "underdog“ (der Somali-Minorität), in dem die Somali-Minorität mit der shifta-Guerilla eine dissoziative Strategie wählte, um ihren Status gegenüber der Kenia-Regierung zu verbessern. Eine assoziative Strategie, also die Kooperation mit der Kenia-Regierung,

\footnotetext{
86 Vgl. Kende (Anmerkung 2)

87 Vgl. Rosenau, James N., Internal War as an International Event, in: Rosenau (Ed), (Anmerkung 7), S. 63.

88 Vgl. Anmerkung 38 .

89 Vgl. Deutsch, Karl W., The Analysis of International Relations, Englewood Cliffs, N. J., 1968, S. 144: "mixed type of war, in which large domestic rebel forces are fighting against the government of the target country in the manner of a genuine civil war, while at the same time receiving substantial and sustained support from at least one outside power. ${ }^{\alpha}$ war, while at the same time receiving substantial and

90 Vgl. dazu Galtung, Johan, The War System, unv. Manuskript; Senghaas, Dieter, Friedensforschung und Dritte Welt, in: Afrika Spectrum, H. 3, 1973, i. E.
} 
hätte möglicherweise den underdog-Status der Somali noch stabilisiert. Durch die Intervention Somalias zugunsten der Somali-Minorität wurde allerdings später eine gewisse Symmetrie des Krieges erreicht, so daß möglicherweise nun für die Somali in Kenia eine assoziative Strategie akzeptabel sein könnte. Wenn die Kenia-Regierung die Somali-Minorität heute auch militärisch unter Kontrolle hat, so scheint das Kernproblem des shifta-Konflikts jedoch weiterhin ungelöst zu sein: das Problem einer Assimilierung der Somali und ihre Integration in den kenianischen Staat. Bei relativ kleinen Minderheiten, die zudem noch territorial zerstreut leben, mag dieses Problem, vorausgesetzt, die Regierung ist guten Willens und verfügt über genügend Zeit, kein so schwieriges sein; anders jedoch bei den Somali, die zahlenmäßig relativ bedeutend sind und territorial geschlossen leben an der Grenze $\mathrm{zu}$ einem Staat, der sie aktiv unterstützt. Nationale Identifikationen sind jedoch keine konstanten Größen; sie sind prinzipiell veränderbar und können durch die bewußte Politik einer Regierung durchaus in eine andere Richtung gelenkt werden. Eine Assimilierung und Integration der Somali könnte der Kenia-Regierung gelingen, wenn sie geduldig die Hindernisse aus dem Weg räumt, die einer territorialen und sozialen Integration im Wege stehen. Dies erforderte vor allem die von einer relativen Autonomie der Somali ihren Ausgang nehmende vorsichtige Heranführung der Somali an den territorialen Nationalismus Kenias und eine sozio-ökonomische Entwicklung mit dem Ziel der Hebung ihres Lebensstandards. Genau dies meinten der Abgeordnete Odinga und der Verteidigungsminister Mungai, als sie im kenianischen Parlament davon sprachen, die Somali in Kenia mit dem Ziel zu „indoktrinieren“ „to understand that they are part and parcel of Kenya"91, und die Regierung Kenias aufforderten, sozial und ökonomisch mehr für die Somali zu tun, um ihnen das Leben in Kenia so attraktiv zu machen, daß sie gar nicht mehr den Wunsch nach einem Anschluß an Somalia verspüren würden ${ }^{92}$. Der Bericht eines die Somali-Gebiete Kenias im Jahre 1970 bereisenden Journalisten deutet zwar auf etliche soziale und ökonomische Verbesserungen für die Somali hin, stellt deren politische Loyalität gegenüber der Kenia-Regierung jedoch weiterhin in Frage ${ }^{93}$. $\mathrm{Zu}$ stürmische Assimilierungsmaßnahmen der Regierung könnten leicht konterproduktiv werden. Ein weiterer wesentlicher Faktor im Zusammenhang mit einer dauerhaften Friedenslösung ist die Haltung der Republik Somalia; die Ereignisse auf der OAU-Tagung vom Mai 1973 deuten auf ein mögliches Wiederaufleben des Grenzkonflikts zwischen Somalia und seinen Nachbarn hin ${ }^{91}$.

\footnotetext{
91 Republic of Kenya, The National Assembly, House of Representatives, Official Report, vol. X, Part II, Fourth Session, 4. November 1966, col. 1727.

92 Republic of Kenya, The National Assembly, House of Representatives, Official Report, vol. IV, Second Session, 26. März 1965, col. 886.

93 Boydell, Michael, Bringing a new way of life to Kenya Somalis, in: East African Standard, 13. Februar 1970.

94 Vgl. dazu Africa Research Bulietin, Mai 1973, 2843-2851.
} 
"Fearless-Proposals" of the Labour Government to the Anglo-Rhodesian agreement of 1971. It is shown that both the Labour and the Conservative governments, which refused to resort to military intervention for enforcing its imperial legal claim, with the application and timing of their "Six Principles" agreed to a settlement that would have brought majority rule in about 100 years' time, according to pessimistic estimates, and even on very optimistic assumptions no sooner than in 50-60 years' time. The Pearce Report documented the rejection of this agreement between two white governments by a overwhelming majority of the Africans. After the failure of international sanctions due to the complicity of white regimes in Southern Africa and international economic interest only the Liberation Movements could defeat the internal colonialism in Rhodesia.

\section{The Shifta-War in Kenya}

\section{By Volker Matthies}

The most immediate and serious security threat to independent Kenya was the guerilla warfare of the Somali shifta fighters in the north-eastern part of the country. Economically and socially neglected by the British colonial administration, the Somali in Kenya were highly suspicious of the central government. Moreover, the Somali were influenced to a high degree by tribal feelings and nationalist emotions, which were based on an ethnic-cultural identity of all Somali people living in the Horn of Africa and which aimed at secession from Kenya and at political union with the neighbouring Republic of Somalia. Thus a growing number of Somali in Kenya felt no strong loyalty to the new state in which they happened to live but instead considerable sympathy with the Somali Republic, and they took their resentment towards the Bantu-dominated Kenya government to the stage of an armed insurgent secessionist movement. Although the shifta warfare was truly locally inspired, it gained increasingly support from the Republic of Somalia. For the government of Kenya the shifta-guerilla was an essentially tribalist movement which threatened the territorial integrity and political sovereignty of the state. Since Kenya lacked internal cohesion and a deeply rooted nationhood, any surrendering to secessionist pressure was dangerous. To allow one etnic or tribal group to secede might lead to secessionist or separationist tendencies elsewhere and might add new problems to the difficult task of nation-building. Thus the government of Kenya has been involved since 1963 on an increasing scale in a costly anti-guerilla warfare to eliminate the shifta threat. After four years of fighting the government was successful in creating military and political control in the Somali area of Kenya.

Theory and Comparative Legal Aspects of Economic Integration among Developing Countries

\section{By ERnst U. Petersmann}

Regional economic integration by means of economic unions, common markets, customs unions, free trade areas, partial preferential agreements or project arrange- 